\title{
Notas
}

\section{Avicultura tradicional en Chiloé (siglo XIX)}

\section{Introducción}

Chiloé es una isla situada a orillas del Pacífico sur, dueña de una cultura exclusiva que fue consolidándose de manera definida desde el tiempo de la colonia española, a la que perteneció desde los tempranos años de 1567. La provincia sufrió un acentuado aislamiento histórico que favoreció la perpetuación de antiguos usos y costumbres. La vida se desarrollaba en un medio rural muy sufrido, y las crónicas insisten en la escasez del metálico y en el ejercicio de intercambiar bienes y servicios habitualmente.

La domesticación de aves ha sido una constante y, naturalmente, los animales de granja tradicionales son, desde siempre, parte de Chiloé. Así por ejemplo dice un sagaz religioso a fines del siglo XVIII refiriéndose a las casas de la gente de Chiloé: «Luego que se entra del umbral de la puerta para dentro está á una vista toda la casa con quanto en ella tienen, y allí se hallan tambien las gallinas, y otros animales domesticos» (González de Agüeros 1791: 111).

\section{Relato de un hombre de mar en el siglo XIX}

Edward Blanckley fue un capitán inglés ${ }^{1}$ que navegó entre 1831 y 1834 por los mares sudamericanos a bordo de la corbeta Pylades (Urban 1845: 82). Dotado de un gran poder de observación e inteligencia, escribió algunas de sus impresiones al pasar por la isla de Chiloé. El marino, al tratar de la historia natural de esta región. dice entusiasmado:

«It abounds in insects, butterflies, and birds, very choice and rare, many not being known in other parts of the continent of South America. I shall mention one curious bird, called in Chiloè canguena, which is only to be found here. I succeeded in procuring four specimens of it, with the hope of introducing the into England. They partake in appearance and formation to the duck, goose, guinea fowl, and, in plumage, of the partridge (red-legged) and pheasant. In size they are nearest the guinea fowl; and although web-footed, they do not take the water, but area constantly dipping their feet in small pools to prevent the web of the feet from cracking. Their legs are black, and the breast is marked like the red-legged partridge. The belly is of a light brown, the back like a hen pheasant; while the neck resembles the guinea fowl, the upper part being marked not unlike that bird. The head, with a black beak, is exactly that of a Bengal goose, but with a remarkably fine eye. When caught young, they are easily do-

\footnotetext{
1 Hijo de H. S. Blanckley, cónsul general en Algiers. Ingresó a la Marina británica como guardia marina en 1805, pasando por distintas embarcaciones y asistiendo a varios enfrentamientos navales, siendo promovido finalmente a teniente en 1815, hasta llegar al grado de comandante en 1825 (Burke 1884: 90; O'Byrne 1849: 89; Urban 1845: 82). Contrajo matrimonio en dos ocasiones: en 1820 con Harriet Matcham y en 1841 con Sarah Elizabeth Nayler (Innes et al. 1827: 125; O’Byrne 1849: 89).
} 
mesticated, and live with other poultry. Their flesh partakes much of the flavour of the pheasant, but not so dry. Those I procured were given me by the Governor, and were taken from his poultry-yard. I lost them one by one during a severe illness on my passage home; in consequence of not being able to attend personally, which I previously did, to see that they had water twice a day to paddle their feet in. This not having been attended to, their webs split, caused sores, and they died in a few hours. What I regretted also was, that, on my recovery, I discovered that, as they died, their skins had been committed to the deep. One I have brought home, but I fear it is not in so perfect a state as to give a just idea of the animal; it is, however, in the hands of a skillful naturalist» (Blanckley 1834: 355-356).

Es decir, describe prolijamente un ave nativa de Chiloé, indicando que se encuentra domesticada y que puede hacérselo con facilidad cuando se crían pichones; describe también su carne (de sabor muy parecido al del faisán); y relata que intentó llevar cuatro ejemplares (que le fueron regalados por el gobernador de Chiloé -que debía ser Juan Felipe Carvallo-, siendo tomados de su propio corral) en su viaje de regreso a Inglaterra. Los individuos murieron durante la navegación, por lo que su idea de introducir a la especie en Inglaterra no tuvo éxito.

El relato aparenta centrarse en un animal de género Chloëphaga sp., que corresponde a cinco especies de aves anseriformes endémicas del continente sudamericano (Martín 1984: 6), cuyos nombres vulgares son las voces canquén (nótese la similtud fonética con el vocablo «canquena», seguramente retocado por el oído inglés), canquín, caiquén o cauquén, semejantes a avutardas. Las mismas (exceptuando Chloëphaga hybrida, Molina 1782) tienen hábitos migratorios, son gregarias, y se juntan en grandes bandadas en la estación no reproductiva; caminan erguidas y tienen formas esbeltas de cuello largo y cabeza pequeña (Palermo 1984: 3), pareciendo gansos de pico corto (Figura 1).

Hay dos especies que pueden ajustarse a la descripción del ave, atendiendo además al nombre mencionado. Por un lado, el caiquén (Chloëphaga picta, Gmelin 1789), una especie de hábitos acuáticos y terrícolas, citada como de fuerte caminar, de dieta estrictamente herbívora, y a quien se puede encontrar en pareja o en grupos familiares de hasta 30 individuos (Giardina 2010: 96). Y, por otro lado, también el llamado canquén o cauquén (Chloëphaga poliocephala, Sclater, P1. 1857), que tiene más dibujos en su plumaje que el anterior, y del que consta que «Su verdadera patria donde anida son las islas de Chiloé, en invierno va más al norte hasta Curicó, en Ancud se vé domesticada» (Philippi 1868: 280). Las dos especies mencionadas migran desde el sur de Chile y Argentina a la provincia de Buenos Aires y los valles del Río Negro y del Colorado, en Argentina, por los meses de mayo a agosto (Pereyra 1943: 85; Palermo 1984: 6).

La diferencia fundamental entre el caiquén (Chloëphaga picta) y el canquén (Chloëphaga poliocephala) es que la segunda de ellas no posee dimorfismo sexual, mientras que sí lo tiene marcadamente la primera. Blanckley menciona, por otro lado, que las patas de las aves son de color negro y las patas de Chloëphaga poliocephala son negras en la parte interna y naranjas en la externa, si bien el macho de Chloëphaga picta presenta también la característica de patas negras. De todas maneras, no es infrecuente encontrar las dos especies juntas en un mismo espacio, mezcladas incluso unas con otras. 
Figura 1: Chloëphaga poliocephala Sclater, Pl. 1857. (Fotografía del autor).

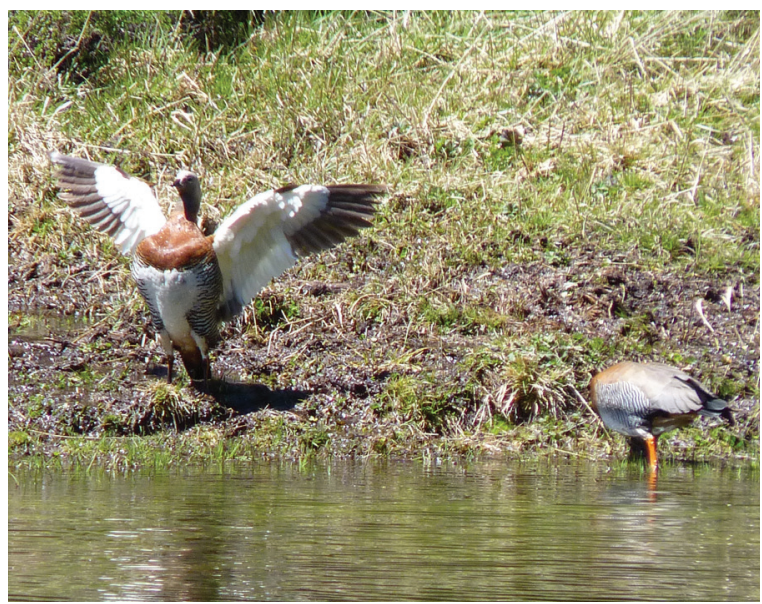

\section{Otras referencias}

Habrán de pasar casi quince años de publicado el dato de Blanckley (1834), para que el naturalista Claudio Gay destine un interesante párrafo a la especie:

«Los Cauquenes se hallan en el estrecho de Magallanes y van á veces ácia el norte hasta el rio Rapel (34 grad. de lat.). Abundan en Chiloe y se ven volar en bandadas de mas de ciento. Se alimentan de yerbas: dañan mucho á los trigos cuando están verdes, y aun comen los granos. La hembra es algo mas pequeña que el macho; pone diez á quince huevos en la orilla de los lagos, entre los juncos ó gramas, iguales á los de las gallinas, muy buscados por los labradores para reunirlos á los de estas cuando empollan: los polluelos que produce los cuida y proteje la gallina como los suyos, pero esos los persiguen algunas veces. Tambien se crian dichas Aves en muchas casas, no solo por la elegancia de sus formas y plumaje sino mas aun por su escelente carne; las alimentan con trigo, mas a causa de su pico no son tan diestras como los pollos, y recojen pocos granos; así están obligadas de recurrir á la yerba, la que picotean todo el dia. Cuando se enfadan echan la cabeza y el pescuezo atrás gritando débilmente y repetidas veces pió, pió. Seria una Ave muy útil para los corrales, pues se domestica fácilmente, y es tan poco tímida que aun en los campos se puede acercarse á ella» (Gay 1847: 444).

El científico destaca la abundancia de la especie en Chiloé, su crianza en «muchas casas», y la calidad de su carne. Aunque su relato esté en realidad mal ubicado taxonómicamente (Philippi y Landbeck 1862: 434, 436), los datos que aporta son valiosos y está claro que deben ser atribuidos a alguna de las dos especies de Chloëphaga $s p$. mencionadas anteriormente.

En 1914, un religioso menciona lo siguiente refiriéndose a Chiloé: «Las aves de corral son los pavos, gansos, patos domésticos, cauquenes, palomas y gallinetas» (Cavada 1914: 51), aunque en su errata el autor indique luego que en vez de cauquenes quiso decir «canqueñes» (Cavada 1914: 446), que en todo caso es uno de los sinónimos vulgares de la especie en Chiloé. Lamentablemente, la noticia no da mayores informaciones, en tanto la conjugación verbal de la frase tampoco permite discernir si se trata del tiempo presente (es decir, el año en que fue publicado el manuscrito) 
o si es una manera elíptica de evocar la cultura tradicional de Chiloé (a manera de representar el conjunto de modos de vida, costumbres y conocimientos, como algo estático referido a un tiempo histórico general), pero alcanza y sobra para despejar la duda de si el ave en cuestión era o no usual en los corrales avícolas de Chiloé.

Finalmente, el arqueólogo y etnólogo Ricardo E. Latcham escribe un interesante párrafo que conviene transcribir:

«No se sabe con seguridad si los antiguos chilenos tenían otras aves domésticas, además de las gallinas, porque sobre este punto callan los cronistas; pero es verosimil que hayan domesticado el pato, del cual existían en el país numerosas variedades distintas a las que se habían domesticado en el Perú. Una de ellas era el cauquén, pato de porte mediano, con plumas de cuatro colores, verdinegro, color canela, blanco y azul morado. Es probable que el pato grande del Perú, también se conociera en las provincias del norte y centro del país; pero nos apresuramos a advertir que esta es simple conjetura, porque en cuanto sabemos no existe ningun documento de la época de la conquista o luego después, que menciona el hecho. Sólo podemos alegar, que un siglo más tarde ambas especies eran abundantes en los corrales de los criollos y naturales; aun cuando más tarde, a semejanza del chilehueque, el cauquén había desaparecido de entre las especies domesticadas, siendo reemplazado por otras de origen europeo» (Latcham 1922: 197).

Para concluir, la certeza sobre la especie de la que escribe Blanckley queda reducida a Chlö̈phaga poliocephala (Sclater, P1. 1857) y a Chloëphaga picta (Gmelin 1789).

La posibilidad de criar y reproducir estas especies ${ }^{2}$ no corresponde únicamente al período contemporáneo, y seguramente el conocimiento previo se obtuvo en Chiloé de las antiguas etnias aborígenes ${ }^{3}$. No es desacertado pensar que se haya transmitido

2 Al parecer, la aptitud de domesticar estas aves alcanza hasta el género incluso, ya que se menciona por ejemplo acerca del piuquén (Chloëphaga melanoptera Eyton, 1838) ya en los tempranos años de 1788: «Su carne es de mucho mejor sabor que la de las pavas; y como se domestica y amansa con facilidad, hay muchos labradores que los crian ya en sus propias haciendas.» (Molina 1788: 291). Otro autor coincide en que «Varias personas han tenido Piuquenes domesticados en los corrales, pero no han empollado en la cautividad», aunque disiente de modo contrario en que «Siendo su carne bastante mala los Piuquenes no se suelen cazar» (Philippi y Landbeck 1862: 431). También la llamada cahue (Chloëphaga hybrida Molina, 1782), que se distingue por encontrarse sin excepción en el litoral rocoso alimentándose únicamente de algas marinas (Martín 1984: 6), donde también vive en pareja y se reproduce (Valenzuela Rojas 2002: 3), es citada como «mui recelosos», aunque igualmente habiéndose «visto un par domesticado en Ancud, pero no sabemos si se reproduce en la domesticidad» (Philippi y Landbeck 1862: 438, 439). La bibliografía indica de todas maneras que, de todas las especies mencionadas, el cauquén común (Chloëphaga picta) es una de las más sociables, acentuándose esa condición luego de la crianza de los pichones (Palermo 1984: 3).

3 Se indica por ejemplo la presencia, junto a cabañas indígenas en Tierra del Fuego, de algunos animales amansados: gaviotas como mascotas llevadas en embarcaciones al viajar (Spencer 1931: 106; Weddell 1827: 183), pichones de cauquenes y gaviotas (Lothrop 1928: 159); aunque lo esporádico de esta práctica y el destino final incierto de las piezas hace imposible demostrarla mediante metodología arqueológica (Mameli y Estévez 2005: 60). Además, la población indígena yámana o yaghan (el pueblo originario del extremo meridional del continente) utilizaba los cauquenes para otros usos, como por ejemplo: los huesos de la pata como tubos sorbedores de agua (Mameli y Estévez 2005: 61), tiras de sus pellejos que conservaban el pulmón como adornos cefálicos (Mameli y Escalera 2005: 62), o reservaban los buches para hacer recipientes para guardar aceite (Palermo 1984: 23); y otras etnias australes como los selk'nam u onas suplementaban su dieta básica de guanaco, cormoranes y patos con cauquenes (Borrero 2007: 30), utilizando también los huesos largos como mangos de leznas en el trabajo en cuero (Palermo 1984: 23). Los yámanas hicieron al menos un esbozo de cría, 
la relación de domesticidad entre el cauquén y el hombre en el bagaje de conocimientos incorporados a la cultura de Chiloé.

El ave también es considerada agorera desde la cultura popular de Chiloé, ya que cuando se siente algún golpe repentino se dice que «alguno va a morir porque cayó el caiquén» (Romo 1987: 14).

\section{Conclusión}

Probablemente, los canquenes hayan sido buscados en la época estival aprovechando el tiempo de cría que además era el momento de muda del plumaje y por ello las aves se encuentran imposibilitadas de volar (Lothrop 1928: 159; Mameli y Estévez 2005: 60; Martín 1984: 10), corriendo tras ellos o utilizando algún artilugio, como una vara con un lazo corredizo, tal como lo hacían los indígenas fueguinos (Lothrop 1928: 157ss; Mameli y Estévez 2005: 59), ya sea acercándose a ellos o en emboscada (Tívoli y Pérez 2009: 855) e incluso mediante el uso del garrote en la caza nocturna (2009: 855-856). Los yámanas instalaban trampas en sitios de pastos tiernos, atractivos para estas aves, que estaban constituidas por cercas de estacas con varias entradas en las que se disponían una serie de lazos corredizos hechos con tientos de cuero (Palermo 1984: 22). Dadas estas características de caza, el peso y la etología, es esperable que los canquenes hayan sido consumidos por el indígena en proporciones medias: es decir, en menor porcentaje que las aves que forman grandes colonias (como los pingüinos o los cormoranes), pero en cantidades significativas (Tívoli y Pérez 2009: 856).

El recurso avícola en una sociedad agrícola-ganadera, forestal y pescadora como la de Chiloé, debió tener cierta importancia, en tanto aportaba la facultad de diversificar la dieta alimenticia de la población en general (huevos y carne eran importantes recursos proteicos en tiempos pasados en una sociedad rural), además de aportar elementos de trueque para una economía rural elemental.

La existencia de canquenes (Chloëphaga poliocephala Sclater, P1., 1857 y Chloëphaga picta Gmelin, 1789) como aves de corral queda reconfirmada en Chiloé al menos hasta casi mediados del siglo XIX, como parte de su composición etnográfica histórica, a través de por lo menos tres autores (Blanckley en 1834, Gay en 1847); se la cita también como domesticada hasta en torno a 1870 (Philippi 1868), y quizá hasta los inicios del siglo XX (Cavada 1914). Todo esto contrasta con otra referencia bibliográfica, que es justamente la que indica que ya en el siglo XVIII el cauquén fue reemplazado como ave de corral por los gansos traídos desde Europa, habiendo «desaparecido de entre las especies domesticadas» (Latcham 1922).

Hoy en día -sin referencias sobre este uso en Chiloé de la manera habitual como lo fue en el pasado- queda planteada la posibilidad de estudiar el valor alternativo de la producción avícola de la especie ${ }^{4}$ y la rentabilidad de la explotación comercial de

\footnotetext{
ya que cuando encontraban pichones los llevaban a veces a sus campamentos, donde los alimentaban hasta que alcanzaban un tamaño adecuado para ser consumidos (Palermo 1984: 23).

4 En la Argentina, por ejemplo, el Centro de Investigación y Tecnología Industrial de Carnes ha realizado ensayos de palatabilidad y de otras propiedades de la carne de Chloëphaga picta, obteniendo un valor prome-
} 
este recurso, a considerar junto a su estatus y medidas de conservación ${ }^{5}$. La opción, distinta de los modelos económicos aceptados usualmente, merece ser estudiada, ya que paralelamente a su aptitud avícola (mayormente desconocida), estas aves poseen características deseables en un aprovechamiento racional (por ejemplo, su número en general alto de individuos, su amplia distribución, una dieta flexible y una gran capacidad reproductiva), y queda abierta además la posibilidad de diversificar otras áreas de la economía rural de Chiloé (por ejemplo, la de las valiosas y regaladas gastronomías tradicionales), contribuyendo de esta manera a la supervivencia de la cultura de una importante región histórica. También el manejo sustentable y racional de humedales naturales puede constituir una alternativa que aumente la multiplicidad productiva en distintos lugares, permitiendo simultáneamente la conservación de especies nativas.

\section{Referencias bibliográficas}

BLANKLEY, Edward

1834 «Account of the Island and Province of Chiloè: Extracted from the Remark Book Kept on Board H.M.S. Pylades 1834». The Journal of the Royal Geographical Society of London 4: 344-361.

BORRERO, Luis Alberto

2007 Los selk'nam (onas), evolución cultural en Tierra del Fuego. Buenos Aires: Talleres Gráficos del S.R.L.

BURKE, Sir Bernard

1884 The General Armory of England, Scotland, Ireland and Wales, Comprising a Registry of Armorial Bearings from the Earliest to the Present Time. Londres: Harrison.

Cavada, Francisco Javier

1914 Chiloé y los chilotes. Santiago: Editorial Universitaria.

Comisión Nacional del Medio Ambiente (CONAMA)

2009 Especies amenazadas de Chile: protejámoslas y evitemos su extinción, vol. 1. Departamento de Protección de los Recursos Naturales. Santiago: Grafhika.

dio de carne desosada de $73 \%$ del peso corporal y verificando sus características organolépticas propias. Se envió también al exterior de este país una muestra de carne con carácter de prueba por parte de un frigorífico privado, produciéndose su aceptación y posterior pedido de $3.000 \mathrm{~kg}$, que nunca fue respondido con satisfacción (Martín 1984: 21).

5 En el marco del Reglamento para la Clasificación de Especies Silvestres de Chile, el canquén colorado (Chloëphaga rubidiceps Sclater, Pl., 1861) fue clasificado en la categoría «En Peligro» (CONAMA 2009: 69). En la Argentina, Chloëphaga rubidiceps es clasificada como «En Peligro Crítico» (López-Lanús et al. 2008: 20,56), mientras que Chloëphaga policephala tiene el status de «Amenazada» (López-Lanús et al. 2008: 23), y Chloëphaga hybrida, Chloëphaga melanoptera y Chloëphaga picta aparecen como «Vulnerables» (LópezLanús et al. 2008: 25, 26 y 28 respectivamente). Paradójicamente, Chloëphaga picta ha sido considerada en la Argentina una especie de problema para la agricultura de las provincias de Buenos Aires y La Pampa por el consumo de cultivos finos, y para la ganadería en la Patagonia por competir con el ganado ovino por las pasturas naturales e implantadas, habiendo sido declarada plaga nacional por primera vez en 1931 (Martín 1984: 6). En las temporadas 1972-1973 y 1973-1974 en Tierra del Fuego se realizaron campañas de recolección de huevos de Chloëphaga picta, estimándose una postura total de 180.000 huevos en 24.000 nidos (Martín 1984: 18). 
GAY, Claudio

1847 Historia física y política de Chile, según documentos adquiridos en esta República durante doce años de residencia en ella y publicada bajo los auspicios del Supremo Gobierno. Zoología. Tomo Primero. París: Imprenta de Maulde y Renou.

GiardinA, Miguel Ángel

2010 El aprovechamiento de la avifauna entre las sociedades cazadoras-recolectoras del sur de Mendoza: un enfoque arqueozoológico. Tesis Doctoral. La Plata: Facultad de Ciencias Naturales y Museo, Universidad Nacional de La Plata.

GonZÁLEZ DE AgüEros, Fr. Francisco

1791 Descripción historial de la provincia y archipielago de Chilóe, en el Reyno de Chile, y Obispado de la Concepción: dedicada á nuestro católico monarca Don Cárlos IV. (que Dios guarde). Madrid: Imprenta de Don Benito Cano.

InNES, Anne, Eliza InNes y María InNEs (eds.)

1827 The Annal Peerage of the British Empire, vol. II. Londres: John Murray Albemarle Street.

LATCHAM, Ricardo Eduardo

1922 Los animales domésticos de la América precolombina, tomo III. Santiago: Imprenta Cervantes.

López Lanús, Bernabé, Pablo Grilli, Adrián S. Di Giacomo, Eugenio E. Coconier y Ricardo BANCHS (eds.)

2008 Categorización de las aves de la Argentina según su estado de conservación. Buenos Aires: Aves Argentinas/AOP y Secretaría de Ambiente y Desarrollo Sustentable de la Nación.

LoTHROP, Samuel K.

1928 The Indians of Tierra del Fuego. Nueva York: Museum of the American Indian Heye Foundation.

MAMELI, Laura y Jordi Estévez EsCALERA

2005 Etnoarqueozoología de aves: el ejemplo del extremo sur americano. Madrid: Consejo Superior de Investigaciones Científicas.

MARTín, Susana I.

1984 «La avutarda magallánica (Chloëphaga picta) en la Patagonia: su ecología, alimentación, densidad y control». IDIA 429-432: 6-24.

MoLINA, Juan Ignacio

1788 Compendio de la Historia Geografica, Natural y Civil del Reyno de Chile, $1^{\mathrm{a}}$ parte. Madrid: Don Antonio de Sancha.

O'BYRne, William R.

1849 A Naval Biographical Dictionary: Comprising the Life and Services of Every Living Officer in Her Majesty's Navy, from the Rank of Admiral of the Fleet to that of Liutenant, Inclusive, Londres: John Murray.

Palermo, Miguel Ángel, ed.

1984 «El cauquén común». Fauna Argentina 46. Buenos Aires: Centro Editor de América Latina, Macchi.

Pereyra, José A.

1943 Nuestras aves. La Plata (Argentina): Comisión Central Honoraria de Parques Provinciales y de Protección a la Fauna y Flora aborigen, Ministerio de Obras Públicas de la Provincia de Buenos Aires. 
PhiLIPPI, Rodolfo Armando

1868 «Catálogo de las aves chilenas existentes en el Museo Nacional de Santiago». Anales de la Universidad de Chile 31 (2): 241-335.

PhILIPPI, Rodolfo Armando y Luis LANDBECK

1862 «Sobre los gansos chilenos». Anales de la Universidad de Chile 21 (5): 425-439.

Romo SÁnchez, Manuel

1987 Aves y plantas en la brujería de Chiloé. Santiago: Editorial Nascimiento.

SPENCER, Walter Baldwin

1931 Spencer's Last Journey, Being the Journal of an Expedition to Tierra del Fuego. Oxford: The Clarendon Press.

Tívoli, Angélica Montserrat y Analía F. Peréz

2009 «Rendimiento económico del cauquén común (Chloephaga picta, Familia Anatidae)», en Arqueología de Patagonia: una mirada desde el último confín, Mónica Salemme et al., eds., tomo II, pp. 813-864. Ushuaia: Editorial Utopías.

URBAN, Silvanus

1845 «Obituary». The Gentleman's Magazine 24 (178): 82.

VALENZuela Rojas, Jorge Andrés

2002 Conducta territorial y alimentaria de la caranca (Chloephaga hybrida Molina, 1782) en el litoral pacífico de Chiloé insular. Tesis de Licenciatura. Valdivia: Escuela de Ciencias Biológicas, Facultad de Ciencias, Universidad Austral de Chile.

WEDDELL, James

1827 A Voyage Towards the South Pole Performed in the Years 1822-1824 Containing an Examination of the Antarctic Sea, to the Seventy-Fourth Degree of Latitude: And a Visit to Tierra del Fuego, with a Particular Account of the Inhabitants... Londres: Longman, Rees, Orme, Brown, and Green.

Pablo A. PÉREZ

PAPerez@europe.com 